\title{
2D Mapping and Boundary Detection Using 2D LIDAR Sensor for Prototyping Autonomous PETIS (Programable Vehicle with Integrated Sensor)
}

\author{
Hanugra Aulia Sidharta*1, Sidharta ${ }^{2}$, Wina Permana Sari ${ }^{3}$ \\ 1,2,3Bina Nusantara Institute of Creative Technology Malang/Computer Science Program \\ hanugra.sidharta@binus.ac.id ${ }^{* 1}$, sidharta@binus.ac.id ${ }^{2}$, winapermana@gmail.com³
}

\begin{abstract}
PETIS (Programable Vehicle with Integrated Sensor) is a research project with goal make a robot that move independently with specific purpose. Due complexity of PETIS, research divide into several important sequence. In this research author focus on sense of sight for PETIS, LIDAR chosen due flexible and comprehensive. There is many LIDAR sensor in marketplace, LDS-01 as one of commercial LIDAR sensor available on market, produced by ROBOTIS as one of lowcost LIDAR sensor. Compare with another sensor that cost more than \$1000, LDS-01 just cost lower than \$500. On this research study focus with LDS-01 sensor reading, include hardware, software connection, and data handling. Based on this research LDS-01 as LIDAR sensor can read obstacle with minimum 29,9 cm and maximal 290,7 cm. Comparing with datasheet LDS-01 should work from $12 \mathrm{~cm}$ through $350 \mathrm{~cm}$.
\end{abstract}

Keywords: LIDAR, LDS-01, ROS, 2D Mapping

\section{Introduction}

Robot as one of human creation, born as blind, deaf, mindless, does not have sensory tools. Without equipped with any sense, robot cannot read surrounding and depend with human help. To able read surrounding environment, understand obstacle, read object distance, robot need equip with sensory sense. With ability detect object and surrounding, robot become more agile to perform various goal and task. There is several method to make robot become enable see with their own ways, such as with camera, ultrasonic sensory, and light sensory [1]

Yin proposed using ultrasonic ranging module, this range finder utilize ultrasonic wave through transducer. While there is obstacle in front of sensor, ultrasonic wave will bounce back to receiver. Processing and analyze handle with Atmel AT89S51 microprocessor, this bulk data use to mapping environment in 2D. With this method robot gain 2D mapping with distance from $40 \mathrm{~cm}$ up to $150 \mathrm{~cm}$. These method drawback is accuracy on further object reading, due sensor sensing limitation reading on 30 degree angle [1].

Other method being used is with utilize camera including CV (Computer Vision) system, with using CV system robot can recognize more complex object and obstacle. Most drawback using CV system is on processing data, to process bulk data need expensive system and costly material. CV system also have difficulties deal with distance ranging, system using camera is lack of depth of field [2].

On Moghadam et all study show used of single planar laser range finder. Single planar laser finder work with manipulate of laser range finder, with advantages accurate and fast to measure over small to long range comparing with ultrasonic module. Disadvantages using single planar laser finder it only provides information about object in sight with laser beam. Object below or above sensor will not scanned [3].

2D LIDAR mapping already penetrate to startup, Doxel as a startup based on California use 2D LIDAR mapping combine with Al for monitoring construction site. Doxel's using tracked tank robot with objective scanning construction project for monitoring construction project. Doxel claim, with using their robot will make labor productivity increase 38 percent. This robot will move autonomously scan entire site, include climb up stair and claimed can scan 30,000 square meters on weekly basis [4].

Currently LIDAR sensor cost is bit pricey, due LIDAR manufacture is just few. This lead developer in California called SCANSE develop low cost 2D LIDAR. Compare with product from major manufactured that cost $\$ 1000$ for 2D LIDAR sensor with distance up to $10 \mathrm{~m}$. Scanse make

Sidharta, H., Sidharta, S., \& Sari, W. (2019). 2D Mapping and boundary detection using 2D LIDAR sensor for prototyping Autonomous PETIS (Programable Vehicle with Integrated Sensor). Kinetik: Game Technology, Information System, Computer Network, Computing, Electronics, and Control, 4(2). doi:http://dx.doi.org/10.22219/kinetik.v4i2.731 
a better system with reading up to $40 \mathrm{~m}$ just cost $\$ 250$. SCANSE sensor utilize new method in time of flight using series of micro pulse. Micro pulse used as a checksum to identify angle incoming and outgoing laser. Comparing with traditional method using series of identical laser pulse. SCANSE publish that new 2D LIDAR sensor will use lower power component that lead on cheaper cost compare with traditional LIDAR that use higher power laser to overcome noise. But with draw back on lower update rate to $500 \mathrm{~Hz}$, meanwhile on traditional LIDAR system can read up to $2.000 \mathrm{~Hz}$ to $10.000 \mathrm{~Hz}$ [5]. These promising startups crowdfunding on www.kickstater.com since April 2016 and launching on November 2016. Unfortunately, in 2018 these products not available in Indonesian marketplace, even on international marketplace SCANSE already discontinue.

PETIS (Programable Vehicle with Integrated Sensor) is a project aim make independent smart vehicle with specific goal. As part of PETIS project, research related with LIDAR sensor is important due LIDAR will act as eyesight of vehicle. LDS-01 chosen due many factor, aside from lowest price LDS-01 eminent due support from ROS community.

\section{Research Method}

\subsection{LIDAR}

LIDAR (Light Detection and Ranging) is sensor that utilize LRF (Laser Range Finder), LIDAR is one of sensor which have characteristic reliable and used in many researches related with robotics. LRF sensor is essential to supporting process reading environment, as an eye to robot [6]. Research related with LIDAR began since 1960 in military context, used to detect submarine from airplane. Successfully work at 1970, since than interest regarding LIDAR gradually increase, from military use shift for industrial benefit. With sensor attached to airplane, LIDAR can be used for mapping, cartography, forest management, city planning, oil and gas exploration. Latest technology of LIDAR used in household appliances, like smart vacuum cleaner that move automatically and independent clearing house without human control [7].

LIDAR work with emitting laser beam for measuring distance from sensor to target, then calculate distance through delay between laser emit and laser bounce back to sensor. Compare with RADAR, operating frequency LIDAR is hundreds of Tera Hertz (light pulse), meanwhile RADAR ten of Giga Hertz (electronic waves) [7].

Generally, data produced by LIDAR stored in form binary, consist of coordinate in 2D/3D and intensity of laser. Due burst of LIDAR data, impact on file size that produced. Ying research show for 3D mapping area on rural area as wide as 2,79 square kilometer produce 7 million 3D point [8], this caused by high precision of LIDAR data. Number of data produced with LIDAR will spike for mapping forest area with more complex object.

\subsection{ToF (Time of Flight)}

Principle Time of Flight is distance can be measuring by emitting a signal of known velocity and measure time taken signal reflect to sensor. Radar and sonar work utilize this concept. Historically only sound waves used on ToF with several disadvantages, such as sound wave is slower compare than another wave, difficult maintaining a narrow beam. To eliminate problem researcher used higher wave, such as : ultrasonic wave, visible or infrared light [9].

Time of Flight measurement can measure using formula on Equation 1. Where $d$ is the distance, $v$ is velocity of the signal and $t$ is time taken signal return to sensor.

$$
d=\frac{v t}{2}
$$

\subsection{LDS-01}

LDS-01 (Laser Distance Sensor) is 2D LIDAR sensor produced by Robotis, LDS-01 have capabilities scanning surrounding 360 degree with help DC motor attached to sensor by rubber belt. Table 1 show LDS-01 data, LDS-01 emit class 1 infrared to ensure safety for human eye sight, with wavelength $(\lambda=785 \mathrm{~nm})$. Optimum distance reading is between $120 \mathrm{~mm}$ through 3500 $\mathrm{mm}$ with scan rate $300 \pm 10 \mathrm{rpm}$. Figure 1 show LDS-01 sensor that contain 3-part, laser range finder, rubber belt, and a DC motor.

LDS-01 read data per one degree, its mean on each 1 cycle rotation will generate 360 data. Data connected through UART (Universal Asynchronous Receiver Transmitter) for RS-232C

KINETIK Vol. 4, No. 2, May 2019: 107-114 
protocol for reading using microcontroller. LDS-01 equipped with UART to USB converter, for communicate using USB.

Table 1. Datasheet LDS-01[10]

\begin{tabular}{cc}
\hline Items & Specifications \\
\hline Operating supply voltage & $5 \mathrm{~V}$ DC $\pm 5 \%$ \\
Light source & Semiconductor Laser \\
Diode $(\lambda=785 \mathrm{~nm})$ \\
LASER safety & IEC60825-1 Class 1 \\
Current consumption & 400mA or less (Rush current 1A) \\
Detection distance & $120 \mathrm{~mm} \sim 3,500 \mathrm{~mm}$ \\
Interface & 3.3V USART $(230,400 \mathrm{bps}) 42$ bytes \\
Ambient Light Resistance & per 6 degrees, Full Duplex option \\
Sampling Rate & 10,000 lux or less \\
Scan Rate & $1.8 \mathrm{kHz}$ \\
Dimensions & $300 \pm 10$ rpm \\
& $69.5(\mathrm{~W}) \times 95.5(\mathrm{D}) \times 39.5(\mathrm{H}) \mathrm{mm}$ \\
\hline
\end{tabular}

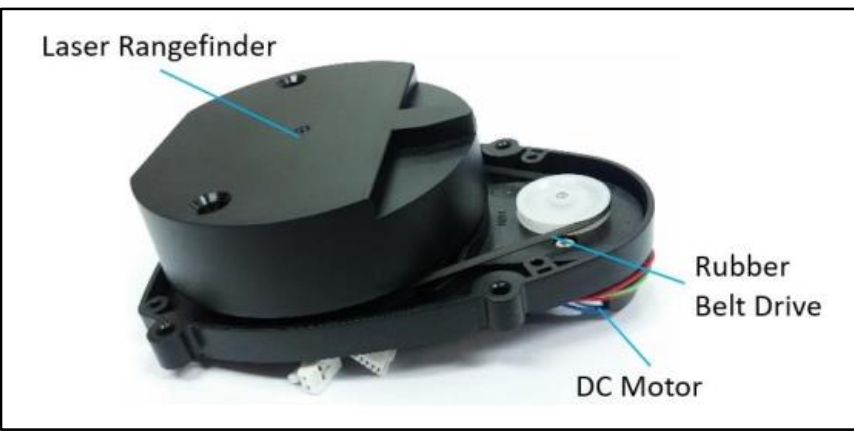

Figure 1. Number of Robot Listed on ROS [11]

\subsection{ROS}

Brief history of ROS, ROS started by Eric Berger during his PhD on Standford University. At that time there is no standard platform infrastructure on robotic research, each robotic researcher creates their own scratch of code. Meanwhile $90 \%$ of their code essentially is identical with same goal, meanwhile $10 \%$ code is novelty on their own respectively. To solve those problem Eric Berger with support professors Ken Salisbury and Andrew Ng started project with name PR1 (Personal Robot). They fundraising called Stanford Personal Robotics Program with goal raise up to US $\$ 4$ million, for hire software engineer supporting ROS [12], [13].

ROS publish first time in 7 November 2007 on Source Forge, set up by Ken Conley, ROS Platform Manager on Willow. ROS evolved from Standford University through Willow Garage and right now become Open Robotics. Since then, number of robots develop using ROS is rising significantly as on Figure 2, and still counting until now. On 2015, first paper related with ROS with title "ROS: An Open-Source Robot Operating System" already cited more than 2020 times. Popularity that paper indicate ROS accepted by many researcher and communities, many robotic startup launch based on ROS, even large and establish companies taking ROS as a partner [11], [14].

With ROS platform, researcher can create embedded system interactive with physical process. Ratasich research combine multiple generic sensor on ROS platform, using multiple and redundant sensor to prevent malfunction or miss reading sensor. He illustrates with height calculation instrument on airplane, attitude reading is vital to airplane pilot. While there is anomaly or faulty on instrument during flight may cause serious trouble, using redundant sensor will help those problem. But using redundant sensor have drawback on addressing problem, using ROS platform help solve this problem [15].

Main advantages using ROS is concept reuse of code, multi programming language capability, high adaptability since its open source. Main obstacle is ROS have lowest score on easy to use criteria, mastering ROS need programming experience and in-depth Operating System knowledge [16]. 
Like Linux evolution, ROS release update twice a year (on April and October), but due user suggestion, since 2013 ROS release once a year and currently release LTS (Long Term Solution) version with latest version is ROS Melodic Morenia $\left(12^{\text {th }}\right.$ version). ROS is a meta operating system, which depend on another Operating System. Due collaborative ubuntu on ROS development, ubuntu distro equipped ported package with ROS. Another common distro used with ROS is Debian or Linux Mint.

With ROS ecosystem, several independent process (called as nodes) organized in directed graph. Each node perform communication using publish and subscribe mechanism that passing message through topic. Node that connected to hardware communicate using device driver. Another node handle calculation and computation subscribe by another node and publish the result [17].

ROS interact between node and software using method as below[18]:

1. Message, each message sent with specific channel, this can be name as topic. This is main method used in communication between node in non-blocking way

2. Service, offering communication between node and act as one to one communication. Include handling handshake process (request, acknowledge, and reply)

3. Action, act as a server node with perform check and standardize each interface with specific task. These functions important to handle complex task that consume amount of time, user can interrupt this request if possible.

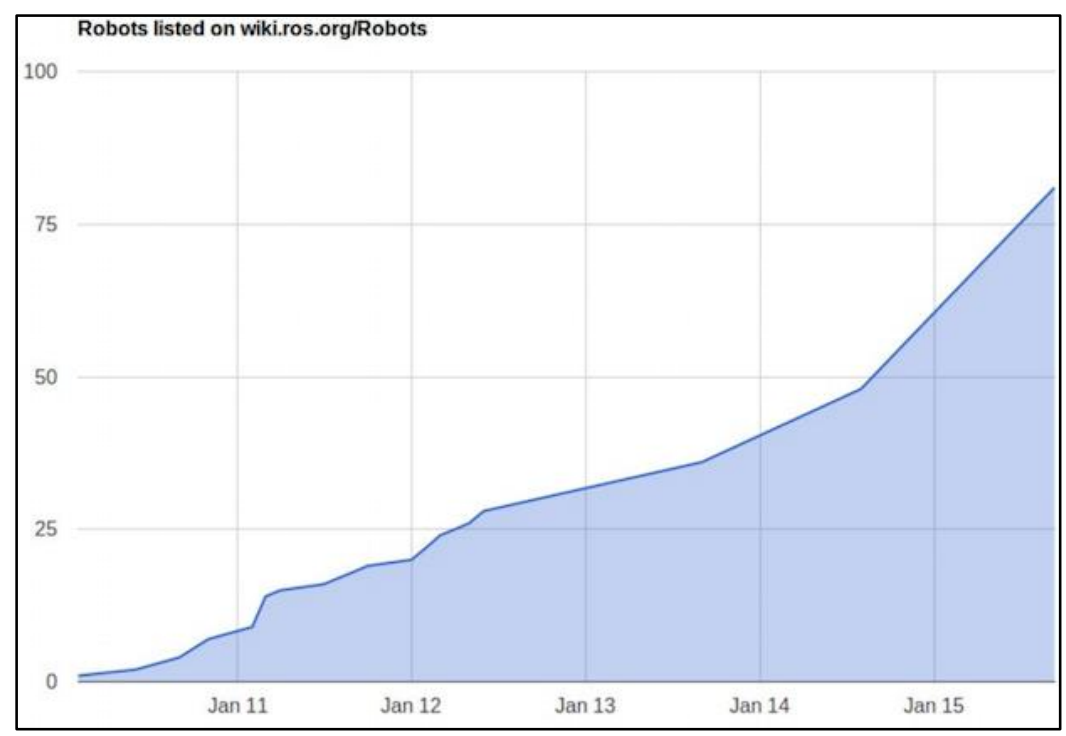

Figure 2. Number of Robot Listed on ROS [11]

Sensor play important role on robot, using sensor robot have capabilities sense imitating human. Some sensor common use with robot such as: geolocation mapping, weather, inertia, gas, vibration, RFID, voice. All those sensors capture and process into information to perform specific goal. There is various sensor used as geolocation mapping supported in ROS, such as LDS (Laser Distance Sensor), LiDAR (Light Detection and Ranging) or LRF (Laser Range Finders), all sensor mentioned is utilize infrared or laser to measure distance between sensor and object. ROS provide environment for develop those sensors, include provide driver on each hardware and library. This can occur due many sensor using same communication protocol, such as I2C or UART [19].

According ROS documentary, sensor can categorize into category, such as:

1. 1D Range Finders : Infrared distance sensor

2. 2D Range Finder : commonly use LDS for mapping environment

3. 3D sensor : sensor with 3D reading capabilities (Intel's RealSense, Microsoft's

Kinect and ASUS's Xction)

4. Audio recognition

5. Cameras

6. Sensor interface

: image processing and object recognition

: another sensor that not use communication through I2C or UART, such as with USB, through internet.

KINETIK Vol. 4, No. 2, May 2019: 107-114 


\subsection{Architecture}

LDS-01 as LIDAR sensor cannot work standalone, it needs connected to several peripheral and software translation. LDS-01 work as active sensor connected to Raspberry Pi 3 with USB, LDS-01 collecting data with 360 degree rotated with DC motor, power for LDS-01 draw from Raspberry Pi 3. Raspberry Pi 3 using Ubuntu 16.04 mate as operating system, ROS act as meta operating system, as intermediate between hardware and software.

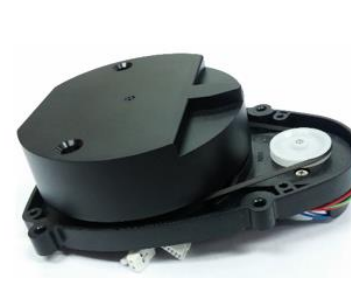

LDS-01

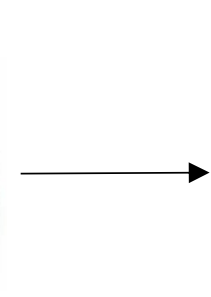

LDS2USB

LDS

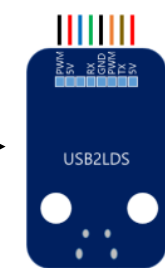

USB

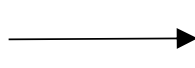

Figure 3. Hardware Architecture

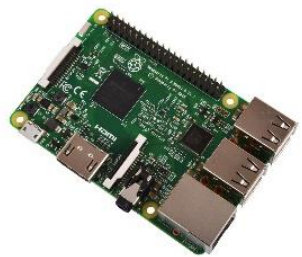

Raspberry Pi 3

Figure 3 shown LDS-01 is connection to Raspberry Pi 3 through LDS2USB, LDS2USB act as converter from serial UART through USB. LDS2USB converter needed to ease data processing, while using Raspberry $\mathrm{Pi}$ more flexible to choose programing language rather than using Arduino or another micro controller.

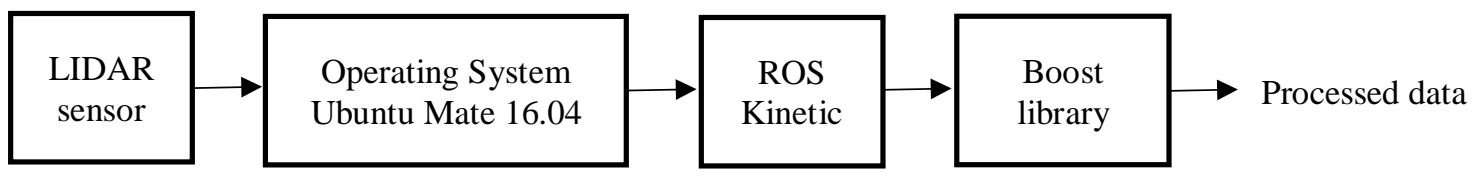

Figure 4. LDS-01 Sensor Data Flow Diagram

In Figure 4 explain LDS-01 connected to Raspberry Pi 3 through one of available USB port, but thanks to ROS provide suitable driver for LDS-01. Connection available through port /dev/ttyUSBO as default port provide by ROS. Ubuntu Mate 16.04 chosen due stable version combined with ROS Kinetic, and due to Raspberry Pi 3 using ARM architecture. Ubuntu Mate 16.04 light enough for LIDAR data capture while ROS Kinetic also running on background.

In this research combine two boost libraries to read LIDAR becoming raw data. Boost.Asio used as communication handler, with feature asynchronous input/output. Boost.Asio provide communication through LDS-01, whether read sensor and give command to sensor. Boost.Array give container to handle serial data produced by LDS-01. LDS-01 produce large quantity of data, when LDS-01 once time revolve it will produce 360 data (one degree will provide one data). Meanwhile based on sensor datasheet, LDS-01 rotate around $300 \pm 10 \mathrm{rpm}$, that can covert to 5 rps (revolution per second). If LDS-01 work scanning data spin for 1 minute with $300 \mathrm{rpm}$, then will produce 108.000 data. Library Boost.Array hold important role as container of those masive data.

\section{Results and Discussion}

Our big goal is creating autonomous vehicle that move freely without human assistance, they can move to fulfill their own objective. To achieve that research topic, need to split into several topic to help focus on research. In this research focus on sensor LIDAR, from hardware setting, communication between hardware and software raw data processing and statistical review.

\subsection{Simulation and testing}

Simulation done in with scenario as below, simulation held on class room with size $5 \mathrm{~m} x$ $5 \mathrm{~m}$. Class room is with empty condition and all object not move in static condition with time frame sample is 5 minutes. Data processing can illustrate on Figure 5 , data scanning from LDS-01 sensor save as to file txt, due output data from LDS-01 is very massive than data must save on compact file. From Table 2 while using txt file format, from experiment with time frame 5-minute 
show data produced with average file size $9,06 \mathrm{MB}$, with minimum file size is $8,93 \mathrm{MB}$ and maximal file size is $9,57 \mathrm{MB}$. For 5 minutes data sampling produced data average $537.296,75$ row of data, in each row of data consist 2 major data, angle and distance (on $\mathrm{mm}$ ), Table 3 show sample data output from LDS-01. Movement each 1 degree produce 1 data consist angle on radius, and distance object measurement in $\mathrm{mm}$ calculate using ToF principle. All distance data produced by LDS-01 in $\mathrm{mm}$, if data shown 0,00 it means there is no obstacle in front of sensor on respectively angle.

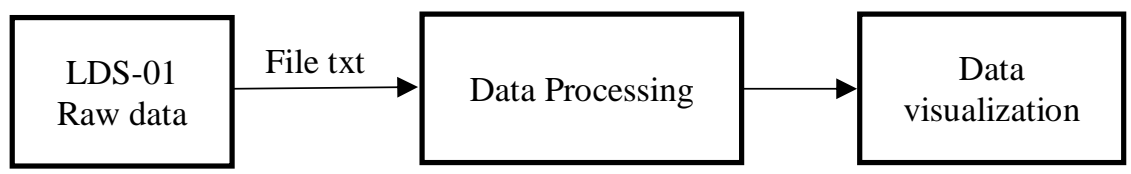

Figure 5. Raw Data Processing Flow Diagram

Table 2. Simulation result based on file size

\begin{tabular}{cccc}
\hline Experiment & File Size (MB) & File Size (Byte) & Number of line \\
\hline 1 & 9,57 & 10.043 .392 & 567.592 \\
2 & 8,94 & 9.383 .936 & 530.323 \\
3 & 9,09 & 9.539 .584 & 539.119 \\
4 & 8,96 & 9.400 .320 & 531.251 \\
5 & 9,02 & 9.461 .760 & 534.721 \\
6 & 9,00 & 9.445 .389 & 533.798 \\
7 & 8,93 & 9.371 .648 & 529.627 \\
8 & 8,97 & 9.412 .621 & 531.943 \\
Average & 9,06 & $9.507 .331,25$ & $537.296,75$ \\
\hline
\end{tabular}

Table 3. Sample Data Output LDS-01 on txt Format

\begin{tabular}{cc}
\hline Angle & Data $(\mathrm{cm})$ \\
\hline $\mathrm{r}[359]$ & 140,00 \\
$\mathrm{r}[358]$ & 108,20 \\
$\mathrm{r}[357]$ & 107,60 \\
$\mathrm{r}[356]$ & 107,40 \\
$\mathrm{r}[355]$ & 0,00 \\
$\mathrm{r}[354]$ & 0,00 \\
$\mathrm{r}[353]$ & 0,00 \\
$\mathrm{r}[352]$ & 0,00 \\
$\mathrm{r}[351]$ & 0,00 \\
$\mathrm{r}[350]$ & 0,00 \\
\hline
\end{tabular}

Processing data done to convert from format Angle and distance through format cartesian coordinate. This done utilize Pythagoras theorem, due known data is angle $\varnothing$ degree and distance data, then coordinate $x$ and $y$ calculation using Equation 2 and 3.

Coordinate calculation plot on Figure 6, this graph produced from LDS-01 by reading based on existing class room. As we can see on Figure 6, there is noise especially on near sensor on coordinate $(0,0)$. Based on LDS-01 datasheet, distance range LDS-01 is $12 \mathrm{~cm}$ to $350 \mathrm{~cm}$, from sensor output found closest reading is $100 \mathrm{~cm}$ and furthest data is $420 \mathrm{~cm}$, while if object scanned is too far will produce data as $0 \mathrm{~cm}$. As shown in mapping plot, there is noise detected surround sensor. To overcome this problem on next research proposed using filtering algorithm EKF (extended Kalman Filter). EKF utilize gaussian noise assumption, with ability predict within uncertainty.

Based on statistical data Figure 7 and Table 4, lower bound is 299 and upper bound is 2907, comparing with datasheet LDS-01, optimal scanning area LDS-01 is from $12 \mathrm{~cm}$ through $250 \mathrm{~cm}$, but from statistical data LDS-01 scanning area is from 19,9 cm through 290,7 cm. Data distributed normal with first quartile $127,7 \mathrm{~cm}$, third quartile $192,9 \mathrm{~cm}$ and median $146,3 \mathrm{~cm}$, scanning span distance is $65,2 \mathrm{~cm}$ based on IQR data.

$$
x=\cos (\Phi) \times \text { distance }
$$




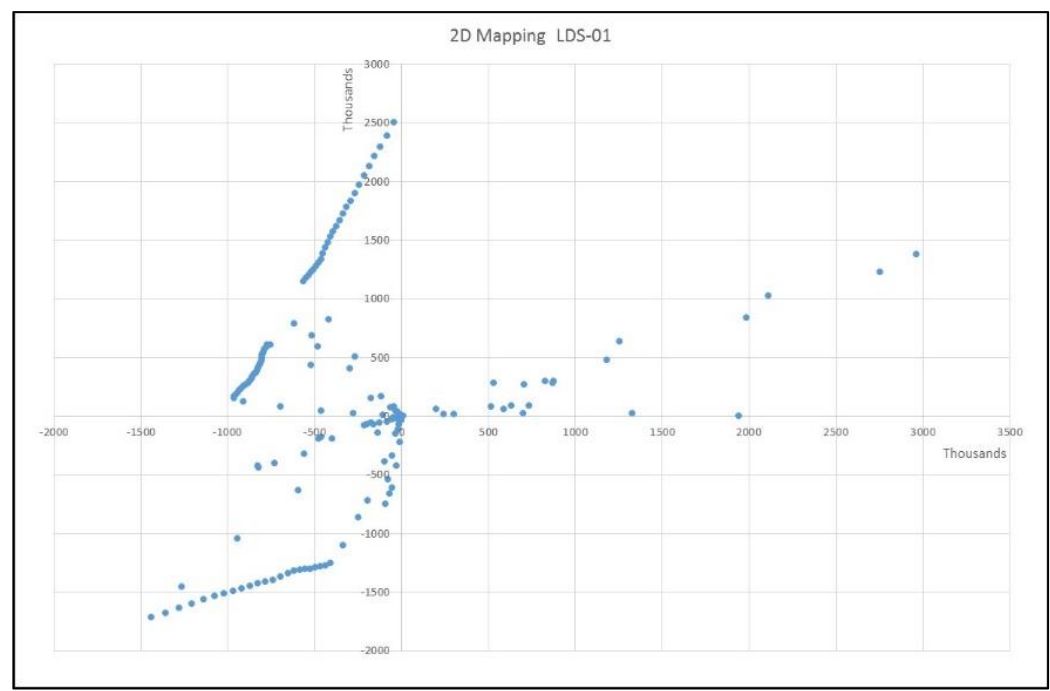

Figure 6. 2D Mapping Data Gathered From LDS-01

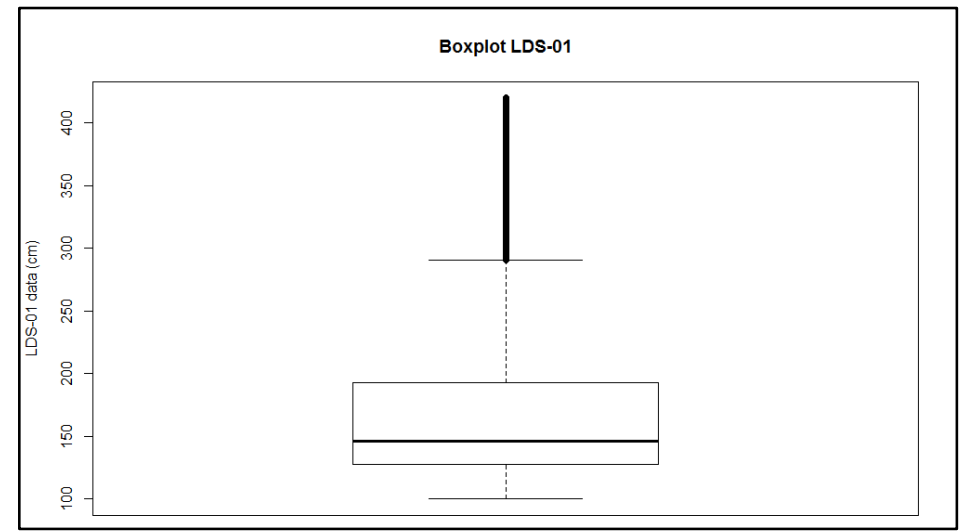

Figure 7. Box Plot Data Sensor Gathered From LDS-01

\begin{tabular}{cc}
\multicolumn{2}{c}{ Table 4. Data Supporting Box Plot } \\
\hline Item & Data \\
\hline Quartil 1 & 1.277 \\
Quartil 2 & 1.463 \\
Quartil 3 & 1.929 \\
IQR & 652 \\
Lower Bound & 299 \\
Upper Bound & 2.907 \\
\hline
\end{tabular}

\section{Conclusion}

Simulation and data processing using LDS-01 is succeed, while data range produced from sensor LIDAR LDS-01 is slightly different. In LDS-01 data sheet, data range from sensor should from $12 \mathrm{~cm}$ to $350 \mathrm{~cm}$, but from simulation found data capture is $29,9 \mathrm{~cm}$ to $290,7 \mathrm{~cm}$. 2D mapping done by convert data through cartesian coordinate, author proposed to use SLAM (Simultaneous Localization and Mapping) algorithm. With using SLAM study related 2D environment sensing become more advanced, algorithm development supported with open source communities.

\section{References}

[1] S. Y. Lee, K. S. Yeoh, and A. Soetedjo, "Developing a blind robot: Study on 2D mapping," Proc. 2008 IEEE Conf. Innov. Technol. Intell. Syst. Ind. Appl. CITISIA, No. July, Pp. 12-14, 2008. 
[2] A. N. Catapang and M. Ramos, "Obstacle Detection using a 2D LIDAR System for an Autonomous Vehicle," no. November, Pp. 25-27, 2016.

[3] P. Moghadam, W. S. Wijesoma, and D. J. Feng, "Improving path planning and mapping based on stereo vision and lidar," 2008 10th Int. Conf. Control. Autom. Robot. Vision, ICARCV 2008, Po. December, Pp. 384-389, 2008.

[4] Evan Ackerman, "Al Startup Using Robots and Lidar to Boost Productivity on Construction Sites," IEEE spectrum, $2018 . \quad$ [Online]. Available: https://spectrum.ieee.org/automaton/robotics/industrial-robots/doxel-ai-startup-using-lidarequipped-robots-on-construction-sites. [Accessed: 05-Dec-2018].

[5] E. Ackerman, "Sweep is a \$250 LIDAR WITH range OF 40 Meters that works Outdoors," IEEE spectrum, $2016 . \quad$ [Online]. Available: https://spectrum.ieee.org/automaton/robotics/robotics-hardware/sweep-lidar-for-robots-anddrones. [Accessed: 05-Dec-2018].

[6] M. Bošnak, "Evolving principal component clustering for 2-D LIDAR data," Pp. 1-6, 2017.

[7] G. Adamo and A. Busacca, "Time of Flight measurements via two LiDAR systems with SiPM and APD," AEIT 2016 - Int. Annu. Conf. Sustain. Dev. Mediterr. Area, Energy ICT Networks Futur., 2016.

[8] D. Lv, X. Ying, Y. Cui, J. Song, K. Qian, and M. Li, "Research on the technology of LIDAR data processing," 1st Int. Conf. Electron. Instrum. Inf. Syst. EIIS 2017, vol. 2018-Janua, pp. $1-5,2018$.

[9] B. M. M. Drayton, "Algorithm and design improvements for indirect time of flight range imaging cameras," Victoria University of Wellington, 2013.

[10] ROBOTIS, "LDS-01 Datasheet," $2018 . \quad$ [Online]. Available: http://emanual.robotis.com/docs/en/platform/turtlebot3/appendix_lds_01/. [Accessed: 08Dec-2018].

[11] B. Gerkey, "ROS, the Robot Operating System, Is Growing Faster Than Ever, Celebrates 8 Years," 920 Dec 2015, 2015. [Online]. Available: https://spectrum.iee.org/automaton/robotics/robotics-software/ros-robot-operating-systemcelebrates-8-years\%0Aspectrum.ieee.org/automaton/robotics/robotics-software/ros-robotoperating-system-celebrates-8-years. [Accessed: 06-Dec-2018].

[12] "1. References As part of the production system for," 2001.

[13] K. Wyrobek, "The Origin Story of ROS, the Linux of Robotics - IEEE Spectrum," IEEE Spectrum Automaton, $2017 . \quad$ [Online]. Available: https://spectrum.ieee.org/automaton/robotics/robotics-software/the-origin-story-of-ros-thelinux-of-robotics. [Accessed: 06-Dec-2018].

[14] E. Ackerman and E. Guizzo, "Wizards of ROS: Willow Garage and the Making of the Robot Operating System," IEEE Spectrum Automaton, 2017. [Online]. Available: https://spectrum.ieee.org/automaton/robotics/robotics-software/wizards-of-ros-willowgarage-and-the-making-of-the-robot-operating-system. [Accessed: 06-Dec-2018].

[15] D. Ratasich, B. Fromel, O. Hoftberger, and R. Grosu, "Generic sensor fusion package for ROS," IEEE Int. Conf. Intell. Robot. Syst., Vol. 2015-Decem, Pp. 286-291, 2015.

[16] W. P. N. Dos Reis and G. S. Bastos, "Multi-Robot Task Allocation Approach Using ROS," Proc. - 12th LARS Lat. Am. Robot. Symp. 3rd SBR Brazilian Robot. Symp. LARS-SBR 2015 - Part Robot. Conf. 2015, Pp. 163-168, 2016.

[17] W. Curran, T. Thornton, B. Arvey, and W. D. Smart, "Evaluating impact in the ROS ecosystem," Proc. - IEEE Int. Conf. Robot. Autom., Vol. 2015-June, No. June, Pp. 62136219, 2015.

[18] M. G. Ocando and N. Certad, "Autonomous 3D mapping of an enviroment, while simultaneously making 2D SLAM , using a single 2D LIDAR and ROS *," Pp. 2-7, 2017.

[19] L. Pyo;YoonSeok, Cho;HanCheol, Jung;RyuWoon, ROS Robot Programing, First Edit. ROBOTIS Co.,Ltd, 2017. 